\title{
Dilinoleoyl-phosphatidic acid mediates reduced IRS-1 tyrosine phosphorylation in rat skeletal muscle cells and mouse muscle
}

\author{
R. Cazzolli • T. W. Mitchell • J. G. Burchfield • \\ D. J. Pedersen • N. Turner • T. J. Biden • \\ C. Schmitz-Peiffer
}

Received: 28 January 2007 / Accepted: 20 April 2007 / Published online: 26 June 2007

(C) Springer-Verlag 2007

\begin{abstract}
Aims/hypothesis Insulin resistance in skeletal muscle is strongly associated with lipid oversupply, but the intracellular metabolites and underlying mechanisms are unclear. We therefore sought to identify the lipid intermediates through which the common unsaturated fatty acid linoleate causes defects in IRS-1 signalling in L6 myotubes and mouse skeletal muscle.

Materials and methods Cells were pre-treated with $1 \mathrm{mmol} / \mathrm{l}$ linoleate for $24 \mathrm{~h}$. Subsequent insulin-stimulated IRS-1 tyrosine phosphorylation and its association with the p85 subunit of phosphatidylinositol 3-kinase were determined by immunoblotting. Intracellular lipid species and protein kinase $\mathrm{C}$ activation were modulated by overexpression of diacylglycerol kinase $\varepsilon$, which preferentially converts unsaturated diacylglycerol into phosphatidic acid, or by inhibition of lysophosphatidic acid acyl transferase with lisofylline, which reduces phosphatidic acid synthesis. Phosphatidic acid species in linoleate-treated cells or muscle from insulin-resistant mice fed a safflower oil-based
\end{abstract}

Electronic supplementary material The online version of this article (doi: 10.1007/s00125-007-0709-x) contains supplementary material, which is available to authorised users.

R. Cazzolli · J. G. Burchfield • D. J. Pedersen · N. Turner •

T. J. Biden $\cdot$ C. Schmitz-Peiffer $(\bowtie)$

Diabetes and Obesity Program,

Garvan Institute of Medical Research,

384 Victoria Street,

Darlinghurst, Sydney, NSW 2010, Australia

e-mail: c.schmitz-peiffer@garvan.org.au

T. W. Mitchell $\cdot$ N. Turner

School of Health Sciences, University of Wollongong,

Wollongong, NSW, Australia high-fat diet that was rich in linoleate were analysed by mass spectrometry.

Results Linoleate pretreatment reduced IRS-1 tyrosine phosphorylation and p85 association. Overexpression of diacylglycerol kinase $\varepsilon$ reversed the activation of protein kinase $\mathrm{C}$ isoforms by linoleate, but paradoxically further diminished IRS-1 tyrosine phosphorylation. Conversely, lisofylline treatment restored IRS-1 phosphorylation. Mass spectrometry indicated that the dilinoleoyl-phosphatidic acid content increased from undetectable levels to almost $20 \%$ of total phosphatidic acid in L6 cells and to $8 \%$ of total in the muscle of mice fed a high-fat diet. Micelles containing dilinoleoyl-phosphatidic acid specifically inhibited IRS-1 tyrosine phosphorylation and glycogen synthesis in L6 cells.

Conclusions/interpretation These data indicate that linoleatederived phosphatidic acid is a novel lipid species that contributes independently of protein kinase C to IRS-1 signalling defects in muscle cells in response to lipid oversupply.

Keywords Diacylglycerol · Fatty acid · Insulin resistance . IRS-1 · L6 muscle cells · Phosphatidic acid .

Protein kinase $C \cdot$ Skeletal muscle
Abbreviations
DAG diacylglycerol
DAGK diacylglycerol kinase
GFP green fluorescent protein
HA haemagglutinin
ipGTT intraperitoneal glucose tolerance test
LPAAT lysophosphatidic acid acyltransferase
nPKC novel protein kinase $\mathrm{C}$
PPAR peroxisome proliferator-activated receptor 
PI3K phosphatidylinositol 3-kinase

PKB protein kinase $\mathrm{B}$

PKC protein kinase $\mathrm{C}$

$\mathrm{PtdH} \quad$ phosphatidic acid

TG triacylglycerol

TLC thin-layer chromatography

\section{Introduction}

Insulin resistance is a reduced capacity of peripheral tissues to respond to physiological levels of circulating insulin and is recognised as a major contributory factor to the development of type 2 diabetes. Insulin resistance in skeletal muscle is particularly significant because of the major role of this tissue in glucose homeostasis [1]; it is strongly correlated with lipid oversupply [2]. The mechanisms by which lipids can generate insulin resistance are therefore an important focus for investigation.

The metabolic and mitogenic actions of insulin are initiated when the hormone binds to its receptor and stimulates a signalling cascade by activation of receptor tyrosine kinase activity and tyrosine phosphorylation of IRSs such as IRS-1 [3]. In contrast, many agents that induce insulin resistance cause phosphorylation of IRS-1 on serine residues, leading to the dissociation of IRS-1 from the insulin receptor, reduced tyrosine phosphorylation and phosphatidylinositol 3-kinase (PI3K) association, and ultimately IRS-1 degradation [4, 5].

Pathophysiological levels of lipid availability have been associated with reduced IRS-1 tyrosine phosphorylation and associated PI3K activity in muscle [6, 7]. It therefore seems likely that the activation of specific lipid-activated signalling pathways that interfere with normal insulin action play a part in the generation of insulin resistance. A link between isoforms of the lipid-activated protein kinase $\mathrm{C}$ (PKC) family and insulin resistance has been reported in several studies [8-12].

While the signalling pathways initiated by NEFA oversupply that are involved in the generation of insulin resistance have been partly elucidated $[6,7]$, less is known about which intermediate lipid species are directly involved. One candidate is the lipid second messenger diacylglycerol (DAG), a potent activator of conventional and novel PKC (nPKC) isoforms, which can be elevated through de novo synthesis from NEFAs. Elevated DAG levels have been demonstrated in rodent and human models of insulin resistance associated with increased lipid availability [8, 10, 13]. A second mechanism, specifically relating to the manner in which saturated lipids can cause insulin resistance, involves generation of the lipid intermediate ceramide [14-16]. Thus different lipid species can interfere with insulin signalling by different means. However, while DAG or ceramide formation is unlikely to account for all lipid-mediated insulin resistance, little is known about other NEFA-derived inhibitors of insulin action. Here, we show that the unsaturated NEFA linoleate, a key NEFA found in diets, causes a reduction in IRS-1 tyrosine phosphorylation in myotubes. While we found no evidence of a role for DAG in this model, we instead identified a novel role for the NEFA-derived second messenger phosphatidic acid ( $\mathrm{PtdH})$, in particular dilinoleoyl-PtdH.

\section{Materials and methods}

Cell culture, fatty acid pre-treatment and insulin stimulation L6 rat skeletal muscle cells were a kind gift from A. Klip (Hospital for Sick Children, Toronto, ON, Canada). Cells were maintained, differentiated and pre-treated with $1 \mathrm{mmol} / 1$ linoleate for $24 \mathrm{~h}$ as previously described [17], with the exception that cells were serum-starved for the final $4 \mathrm{~h}$ of treatment. Cells were stimulated with $100 \mathrm{nmol} / \mathrm{l}$ insulin for $10 \mathrm{~min}$. Glycogen synthesis was measured as previously [17].

Generation and use of recombinant adenovirus and immunoblot analyses These were carried out as previously described [14, 15, 18]. Further details are given in the electronic supplementary material (ESM).

Lipid determination by thin layer chromatography and mass spectrometry Cellular DAG was measured by a method adapted from that described previously by Nakamura and Handa [19]. For the investigation of lipid compartmentalisation, cell homogenates were subjected to sucrose gradient flotation [20, 21]. Phospholipids were separated by two-dimensional thin-layer chromatography (TLC) [22]. Electrospray ionisation mass spectrometry was performed on a Waters Quattro Micro equipped with an electrospray ion source and controlled by Micromass Masslynx version 4.0 software (Waters, Manchester, UK). Detailed methods are given in the ESM.

Comparison of phosphatidic acid species in muscle from mice fed chow or a high-fat diet Glucose intolerance in mice fed a high-fat diet [23] was confirmed by intraperitoneal glucose tolerance test (ipGTT) [24]. Lipid extracts of quadriceps muscles were subjected to mass spectrometry. For further details, see ESM.

Phosphatidic acid treatment PtdH-containing micelles were generated using either dipalmitoyl-PtdH (16:0/16:0), palmitoyl-oleoyl-PtdH (16:0/18:1), palmitoyl-linoleoyl- 
a IP: IRS-1

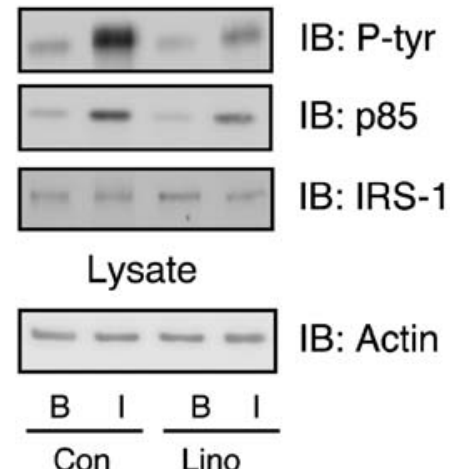

Fig. 1 Effect of linoleate pre-treatment of L6 myotubes on IRS-1 tyrosine phosphorylation and association with PI3K. L6 myotubes were treated without (control, Con) or with $1 \mathrm{mmol} / \mathrm{l}$ linoleate (Lino) for $24 \mathrm{~h}$, including a $4 \mathrm{~h}$ serum-free period, then maintained in the basal state (B) or stimulated with $100 \mathrm{nmol} / \mathrm{l}$ insulin (I) for $10 \mathrm{~min}$ and lysates prepared. a Immunoprecipitates (IP) of IRS-1 were probed for

PtdH (16:0/18:2) or dilinoleoyl-PtdH (18:2/18:2) (Avanti, Alabaster, AL, USA), together with a $4: 1$ excess of phosphatidylcholine (Sigma Chemical, St Louis, MO, USA). Lipids in 2:1 (vol./vol.) chloroform:methanol were mixed, dried under nitrogen and sonicated into $500 \mu \mathrm{l}$ serum-free medium. This suspension was diluted 25 -fold into minimum essential medium $\alpha$ (containing $2 \%$ fetal calf serum [vol./vol.] or serum-free) to give a PtdH concentration of $100 \mu \mathrm{mol} / \mathrm{l}$. L6 myotubes were treated with PtdHcontaining medium for $16 \mathrm{~h}$ in the presence of fetal calf serum, followed by $4 \mathrm{~h}$ in the absence of serum. Cells were stimulated with insulin and harvested as above.

Statistical analysis Results were analysed by Student's $t$ test or ANOVA using Statview 4.5 for Macintosh (available from http://www.statview.com, last accessed in May 2007). Results are expressed as means \pm standard error, with differences considered statistically significant at $p<0.05$.

\section{Results}

Linoleate pre-treatment of L6 myotubes reduces insulinstimulated IRS-1 tyrosine phosphorylation We had previously shown that pre-treatment of myotubes with common nutrient-derived NEFA species caused diminished insulinstimulated glycogen synthesis and that in the case of the saturated NEFA, palmitate, this involved inhibition of insulin signalling at the level of protein kinase B (PKB; also known as Akt) [14]. This was not the case for the unsaturated NEFAs, oleate and linoleate, which appeared to act more proximally to the insulin receptor, and we now wished to determine whether unsaturated NEFA acted at the level of IRS-1. L6 myotubes were pre-treated with linoleate c

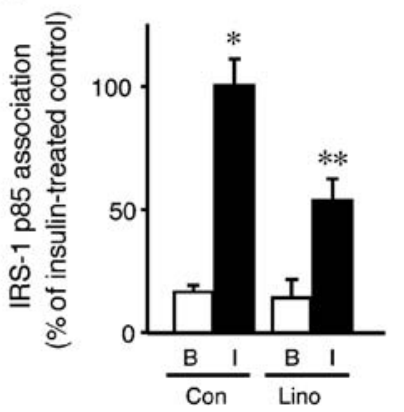

d

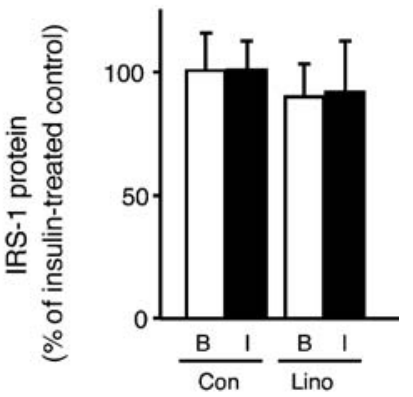

phosphotyrosine (P-tyr), p85 and total IRS-1 by immunoblotting (IB). Lysates were probed for actin as loading control. Combined densitometric data for phosphotyrosine (b), PI3K (c) and total IRS-1 protein (d) from seven independent experiments. $* p<0.001$ for insulin-stimulated vs basal control $\left(t\right.$ test); ${ }^{* *} p<0.02,{ }^{* * *} p<0.001$ for insulin-stimulated linoleate-treated vs insulin-stimulated control

for $24 \mathrm{~h}$, stimulated with insulin and IRS-1 immunoprecipitated from cell lysates. In control cells insulin caused an increase in IRS-1 tyrosine phosphorylation, which was significantly reduced by prior exposure to linoleate (Fig. 1a,b). Linoleate pre-treatment similarly reduced insulin-stimulated recruitment of the p85 subunit of PI3K to IRS-1 (Fig. 1a, c). These effects occurred in the absence of any changes in insulin receptor levels or phosphorylation (data not shown) or in IRS-1 protein levels (Fig. 1a, d). Phospho-specific antibodies against IRS-1 Ser307 and Ser612 $[4,5]$ failed to detect phospho-IRS-1 in this system. Furthermore, whereas insulin alone induced a mobility shift in IRS-1 that was indicative of serine phosphorylation, we did not observe a further mobility shift due to linoleate treatment (Fig. 1a).

Evidence that PtdH, rather than DAG, mediates linoleateinduced IRS-1 depletion Increased PKC activity has been observed in human, animal and cellular models of lipidinduced insulin resistance, including cultured myotubes [18], consistent with a role for activation of PKC by increased intracellular DAG levels. To determine whether exposure of L6 cells to linoleate increased total intracellular DAG, we employed TLC to compare DAG levels directly in control and lipid-treated L6 myotubes. The presence of NEFAs did not raise the levels of DAG, although triacylgycerol (TG) levels were increased more than sixfold (Fig. 2a-c). PKC activation in myotubes by unsaturated NEFA treatment therefore probably involves an increase in a quantitatively minor pool of DAG species that is not apparent at the level of total DAG mass. To examine whether such a pool could be identified upon subcellular fractionation, we subjected myotube homogenates to a well characterised sucrose gradient flotation protocol [20, 21] prior to TLC. While TG was poorly detected in fractions 
from control cells, it was readily observed in the 5 to $10 \%$ sucrose fractions from lipid-treated cells (Fig. 2d,), probably corresponding to TG storage in lipid bodies after de novo synthesis in the endoplasmic reticulum [25]. In contrast, DAG was recovered to an equal extent in the higher sucrose density fractions, corresponding to plasma membrane and endoplasmic reticulum [20, 21], from both control and linoleate-treated myotubes (Fig. 2d). These results are consistent with those obtained using whole extracts, in that TG but not DAG levels are elevated by linoleate treatment. While they also indicate a difference in compartmentalisation between DAG and TG, we were unable to demonstrate the presence of different DAG pools in this manner, further supporting a role for relatively minor species in PKC activation.

To determine the importance of such DAG species in our model, we examined the effects of diacylglycerol kinase (DAGK) $\varepsilon$ overexpression. This isoform of DAGK preferentially catalyses the conversion of DAG comprising polyunsaturated acyl chains to $\mathrm{PtdH}$, and hence promotes the termination of PKC activation [26]. While endogenous expression of DAGKe in skeletal muscle has not been reported, we employed the enzyme as a means to diminish signalling species of DAG and to suppress PKC function. L6 cells overexpressing DAGKe or green fluorescent protein (GFP) as control were pre-treated with linoleate. In control cells this caused chronic activation of nPKC isoforms. This was evidenced by translocation of $\mathrm{nPKC} \varepsilon$ from a cytosolic to a membrane fraction and by the downregulation of cytosolic $\mathrm{nPKC} \delta$, also indicative of chronic activation (Fig. 2e). While nPKC $\theta$ has been implicated in muscle insulin resistance caused by lipid infusion [9], it is poorly expressed in myotubes $[18,27]$ and its overexpression does not inhibit insulin action in these cells [18]. This isoform was therefore not addressed in the present study. Linoleate had minimal effects on the conventional $\mathrm{PKC}$ isoforms, $\mathrm{PKC} \alpha$ and $\mathrm{PKC} \beta$ (Fig. 2e). Importantly, DAGKe overexpression inhibited the chronic activation of $\mathrm{nPKC} \varepsilon$ by linoleate, as indicated by its cytosolic localisation, and also reduced the downregulation of $\mathrm{nPKC} \delta$ (Fig. 2e). These results demonstrate that DAGKe overexpression is a useful tool for preventing the chronic activation of $\mathrm{nPKC}$ isoforms in myotubes by lipid pre-treatment.

We then examined the effect of DAGKe overexpression, and hence inactivation of nPKC, on IRS-1 tyrosine phosphorylation in linoleate-treated myotubes co-expressing haemagglutinin (HA) tagged IRS-1 as a reporter. In agreement with our findings for endogenous IRS-1 (Fig. 1), linoleate reduced HA-tagged IRS-1 tyrosine phosphorylation in control cells co-expressing GFP (Fig. 3a, b). Surprisingly, DAGKe expression alone was sufficient to inhibit insulin-stimulated IRS-1 tyrosine phosphorylation. Furthermore, the presence of DAGKE did not reverse the effects of

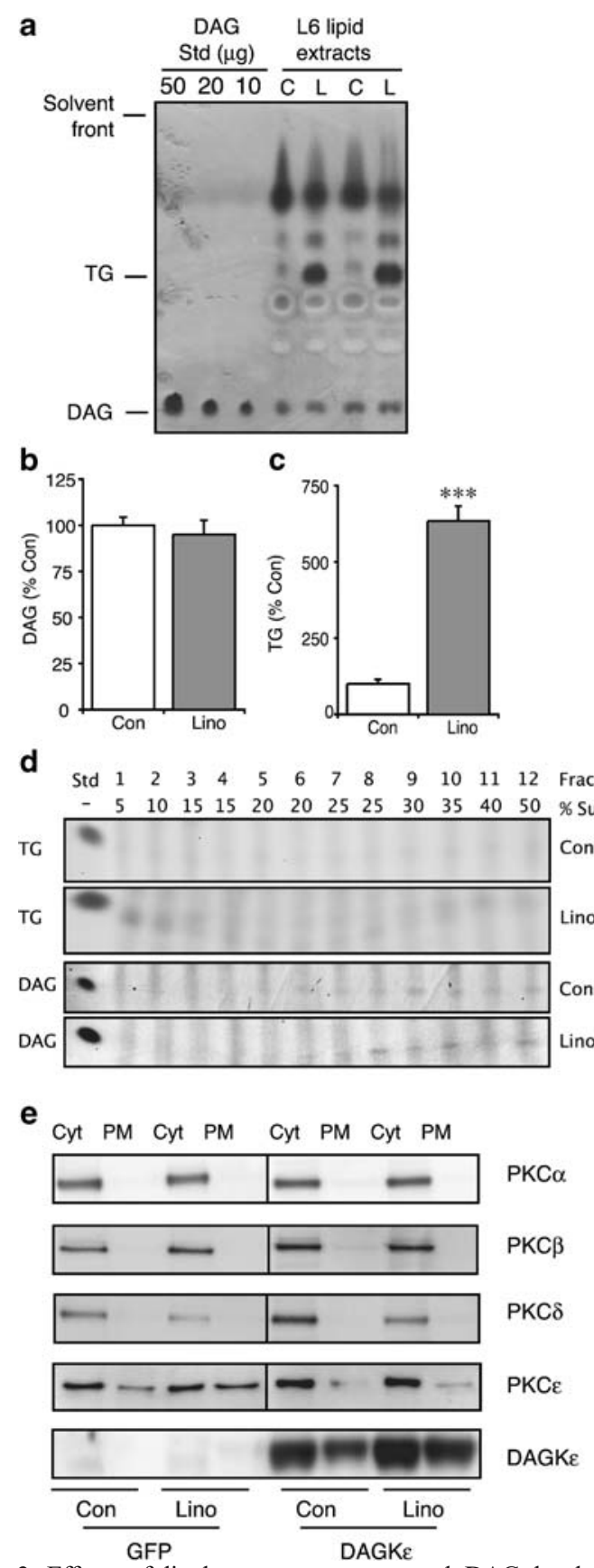

Fig. 2 Effects of linoleate treatment on total DAG levels and PKC isoforms. a L6 myotubes were incubated in the absence (control, C) or presence of $1 \mathrm{mmol} / 1$ linoleate $(\mathrm{L})$ for $24 \mathrm{~h}$ and Folch extracts of total lipids prepared. Neutral lipids were separated by TLC and visualised by Coomassie staining. DAG and TG were identified by co-migration of authentic standards (Std). Combined densitometric analysis of total DAG (b) and TG (c) levels was performed from three independent experiments carried out in duplicate. ${ }^{* * *} p<0.001$ for linoleate-treated (Lino) vs control (Con). d Homogenates of L6 myotubes treated as in a were resolved on sucrose density gradients. Folch extracts of fractions were prepared and analysed by TLC and Coomassie staining. Fraction numbers and corresponding sucrose densities are indicated. Results shown are from one experiment typical of three. e Myotubes were infected with adenovirus for the expression of either GFP or DAGKe. After $6 \mathrm{~h}$, the cells were treated for $24 \mathrm{~h}$ without or with $1 \mathrm{mmol} / \mathrm{L}$ Lino. Cells were fractionated and the cytosolic (Cyt) and plasma membrane (PM) fractions immunoblotted for the PKC isoforms indicated and for DAGKe 
linoleate on IRS-1 tyrosine phosphorylation, in contrast to its effects on nPKCs. Indeed, tyrosine phosphorylation was further reduced when cells were subjected to both DAGKe overexpression and linoleate treatment (Fig. 3a,b). The effects of DAGKE were partly explained by a reduction in IRS-1 protein levels (Fig. 3a,c). These data are not consistent with a role for polyunsaturated DAG and DAGsensitive nPKCs in mediating the inhibition of IRS-1 signalling by linoleate in these cells. Rather, the defects induced by overexpression of DAGKe suggest that the products of this enzyme, polyunsaturated PtdH species, themselves play an inhibitory role.

a
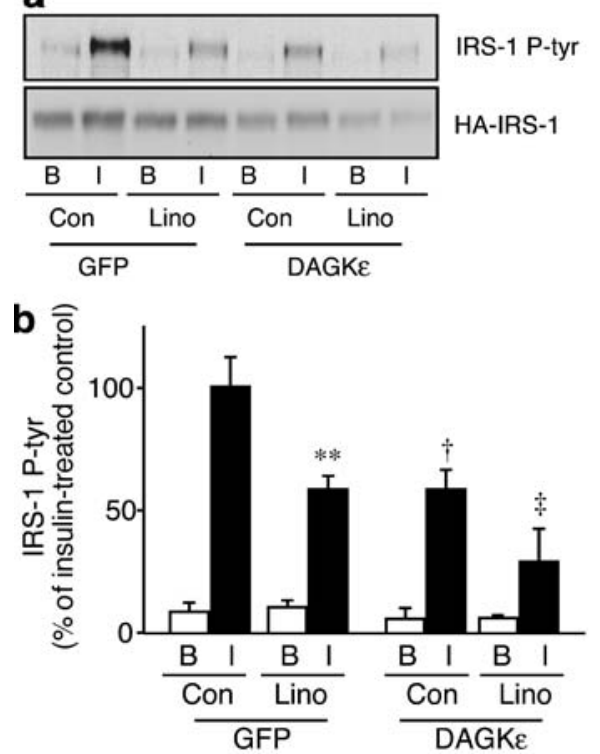

C

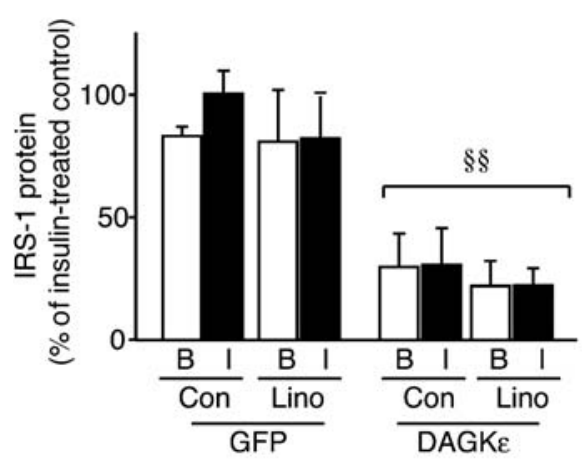

Fig. 3 The effects of DAGKe and lisofylline on IRS-1 tyrosine phosphorylation. a Cells were infected with adenovirus for expression of HA-tagged IRS-1 and either GFP or DAGKe, treated for $24 \mathrm{~h}$ without (control, Con) or with $1 \mathrm{mmol} / 1$ linoleate (Lino) and stimulated with insulin (I). Cell lysates were immunoblotted for phosphotyrosine (P-tyr) and HA-tagged IRS-1. Densitometric analysis of the means of phosphotyrosine (b) and HA (c) is shown for three experiments carried out in duplicate. ${ }^{* *} p<0.01$ for GFP-infected linoleate-treated vs control; $\uparrow p<0.01$ for DAGKE-infected control vs

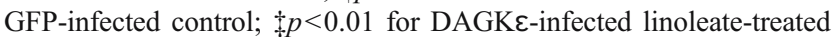

To address this unexpected possibility, we employed lisofylline, an inhibitor of lysophosphatidic acid acyltransferase (LPAAT). LPAAT catalyses the formation of PtdH from lyso-PtdH and acyl-CoA, so its inhibition would be expected to reduce $\mathrm{PtdH}$ formation from linoleate. The presence of lisofylline had reciprocal effects compared with DAGKE overexpression, in that the inhibitor restored insulin-stimulated IRS-1 tyrosine phosphorylation in linoleate-pretreated myotubes and also enhanced total IRS-1 levels (Fig. 3d-f). These results are again consistent with a role for PtdH in the defective IRS-1 tyrosine phosphorylation induced by linoleate.
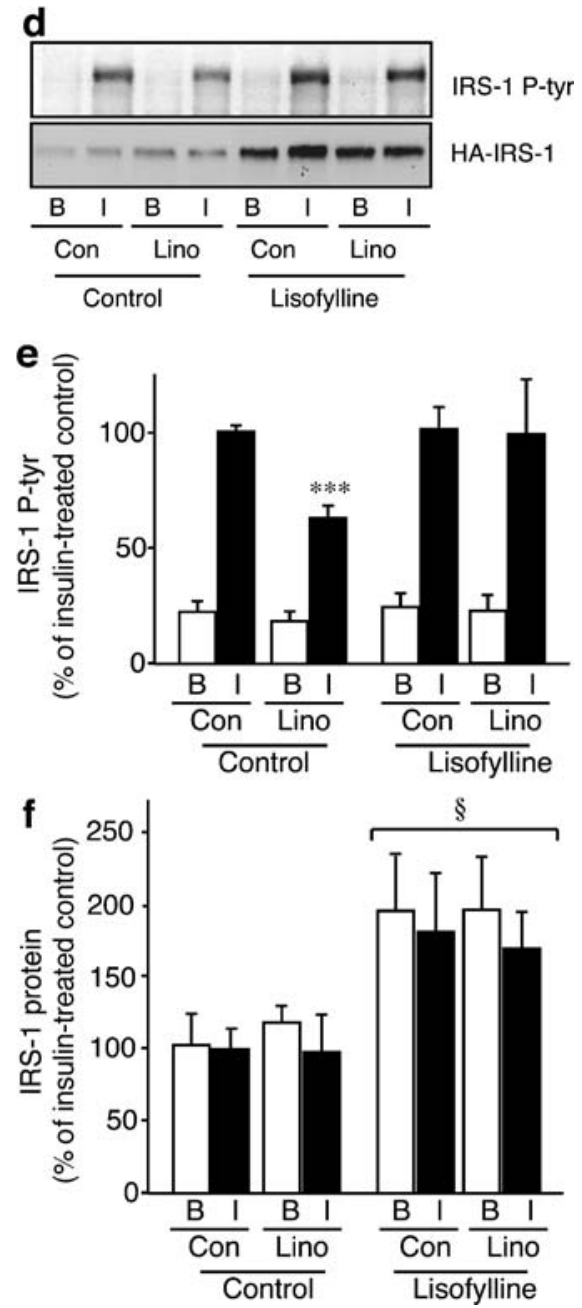

vs DAGKE-infected control (all $t$ test). $\S \S p<0.001$ ANOVA for DAGK $\varepsilon$-infected vs GFP-infected. d Cells were infected with adenovirus for expression of HA-tagged IRS-1, treated for $24 \mathrm{~h}$ with or without $1 \mathrm{mmol} / 1$ linoleate in the absence or presence of $100 \mu \mathrm{mol} /$ 1 lisofylline and stimulated with insulin. Cells lysates were immunoblotted for phosphotyrosine and HA-tagged IRS-1. Densitometric analysis of the means of phosphotyrosine (e) and HA (f) is shown for six experiments. ${ }^{* * *} p<0.005$ for linoleate-treated vs control ( $t$ test). ${ }^{s} p<0.01$ for lisofylline-treated vs untreated (ANOVA). B, basal 
Linoleate treatment increases turnover of cellular PtdH Next we examined whether linoleate pre-treatment affected $\mathrm{PtdH}$ metabolism in myotubes. Intracellular $\mathrm{PtdH}$ levels are extremely low in comparison with DAG and cannot be visualised by Coomassie staining. In a preliminary experiment, L6 cells were instead labelled with $\left[{ }^{32} \mathrm{P}\right]$ orthophosphate, treated with linoleate for up to $24 \mathrm{~h}$ and lipid extracts analysed by two-dimensional TLC. After $4 \mathrm{~h}$ treatment with linoleate, we observed an increase in intensity of a spot that corresponded to $\mathrm{PtdH}$ by reference to a map of authentic standards (Fig. 4a, b; Spot 2). This increase was maintained for at least $12 \mathrm{~h}$ (Fig. 4c). While indicative of changes in $\mathrm{PtdH}$ turnover rather than of total PtdH levels, these results suggest that linoleate induces alterations in $\mathrm{PtdH}$ metabolism.

Linoleate induces the appearance of specific PtdH species To expand on this finding, we determined whether linoleate induced changes in the molecular species present in the $\mathrm{PtdH}$ pool. Mass spectrometry was performed on lipid extracts from L6 cells, treated for either 6 or $24 \mathrm{~h}$ with linoleate. Representative mass spectra are shown (Fig. 4d, e) and the relative abundance of $\mathrm{PtdH}$ species are detailed in Table 1. The greatest differences between control and linoleate-treated myotubes were in the appearance of linoleoyl-containing $\mathrm{PtdH}$. Thus forms of $\mathrm{PtdH}$ containing palmitoyl/linoleoyl (16:0/18:2), stearoyl/linoleoyl (18:0/ 18:2), oleoyl/linoleoyl (18:1/18:2) and dilinoleoyl (18:2/ 18:2) were not detected in control cells, yet after $6 \mathrm{~h}$ linoleate treatment, each accounted for between 6 and $14 \%$ of the total $\mathrm{PtdH}$ (Table 1 ). In each case, the relative abundance was even greater after $24 \mathrm{~h}$ (Table 1). These results confirm that a remodelling of $\mathrm{PtdH}$ species occurs in linoleatetreated L6 cells.

Fig. 4 Effect of linoleate on the phospholipid pool of L6 myotubes. Cells were labelled for $24 \mathrm{~h}$ with $\left[{ }^{32} \mathrm{P}\right]$ orthophosphate and then treated without (control) (a) or with (b) $1 \mathrm{mmol} / 1$ linoleate for up to $24 \mathrm{~h}$. Lipid extracts from cells were subjected to two-dimensional TLC. Phospholipid separations from cells treated for $4 \mathrm{~h}$ are shown. Spots were identified by comparison to authentic standards: Origin; 1, cardiolipin; 2, PtdH; 3, phosphatidylglycerol; 4, phosphatidylethanolamine; 5, phosphatidylserine; 6 , phosphatidylinositol; 7, phosphatidylcholine; 8 , sphingomyelin. c Densitometric analysis of label incorporation into phosphatidylethanolamine (open circles) and PtdH (filled circles) from one experiment. d, e Representative glycerophosphate (mass to charge ratio $[\mathrm{m} / \mathrm{z}] 153.0)$ precursor ion spectra showing the profile of L6 rat skeletal muscle cell phospholipids within the $m / z$ range $640-710$. Cells were incubated for $6 \mathrm{~h}$ in the absence (control) (d) or presence of linoleate (e). Ions in the mass spectrum from linoleate-treated cells have only been labelled where they differ from control. Ions present at $\mathrm{m} / \mathrm{z}$ 685, 686, 698 and 700 do not correspond to any PtdH species as confirmed by tandem mass spectrometry (data not shown). The representative spectra shown are from lipid extracts that did not contain the internal standard (PtdH 17:0/17:0). Values in each spectrum have been normalised to the most abundant ion
Elevation of 18:2/18:2-PtdH in skeletal muscle from mice fed a high-fat diet Because linoleate induced the appearance of highly novel PtdH species in L6 myotubes, we wished to confirm whether these alterations occurred in vivo in skeletal muscle rendered insulin-resistant through increased lipid availability. We therefore examined $\mathrm{PtdH}$ species in lipid extracts of quadriceps muscle obtained from mice fed either a high-fat diet based on safflower oil, which has a high linoleate content (approximately $80 \%$ ), or mice on a standard chow diet as control. The effect of a safflower oil-based high-fat diet on glucose disposal in
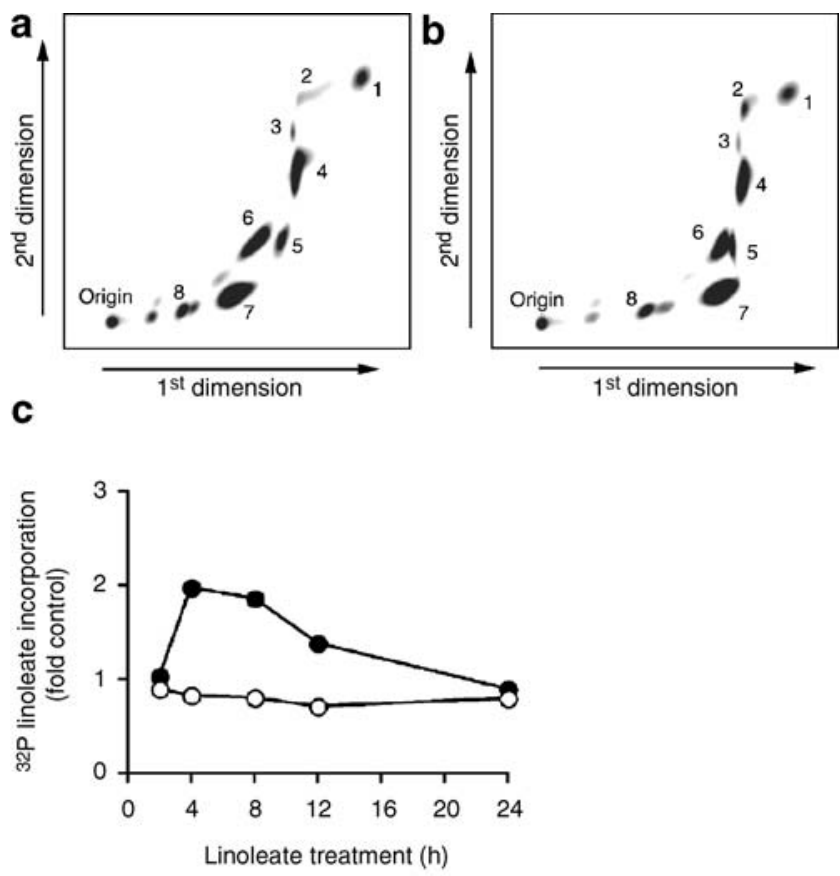

d
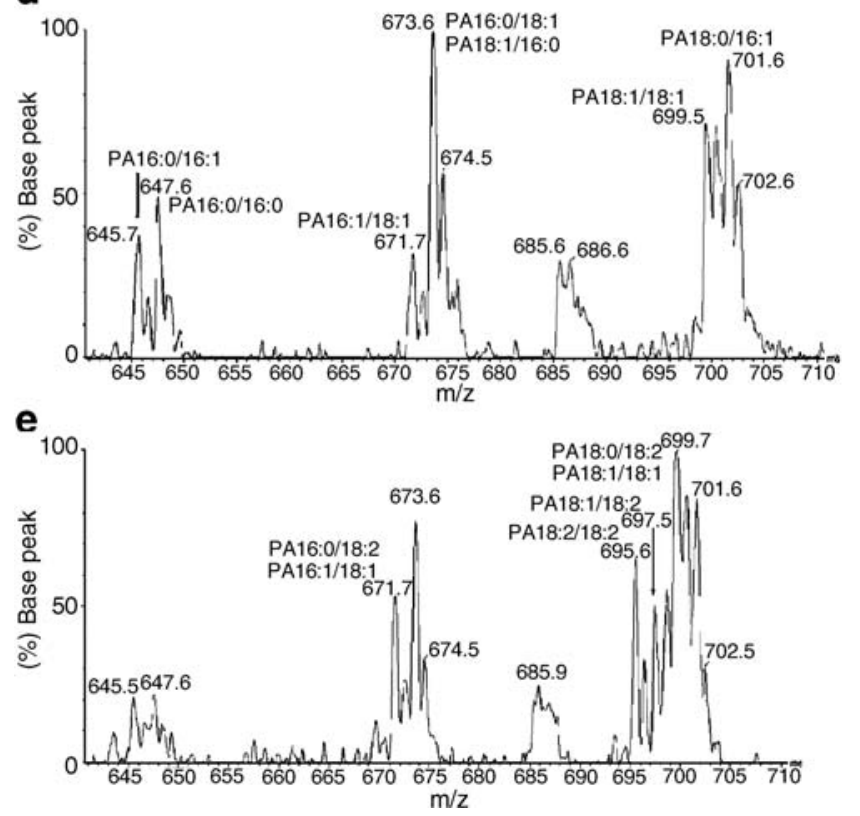
Table 1 Relative abundance of PtdH molecular species in L6 myotubes pretreated in the absence or presence of linoleate

\begin{tabular}{llllll}
\hline$m / z$ & Acyl composition & $6 \mathrm{~h}$, control $(\%)$ & $6 \mathrm{~h}$, linoleate $(\%)$ & $24 \mathrm{~h}$, control (\%) & $24 \mathrm{~h}$, linoleate $(\%)$ \\
\hline 645 & $16: 0 / 16: 1$ & 11.9 & 6.1 & 14.9 & 3.0 \\
647 & $16: 0 / 16: 0$ & 12.2 & 3.5 & 7.0 & 2.1 \\
671 & $16: 1 / 18: 1$ & 9.8 & 6.1 & 12.4 & 4.2 \\
& $16: 0 / 18: 2$ & 0.0 & 6.2 & 0.0 & 12.6 \\
673 & $16: 0 / 18: 1$ & 12.1 & 9.4 & 15.9 & 5.1 \\
& $16: 1 / 18: 0 /$ & 16.0 & 7.5 & 0.0 & 5.2 \\
695 & $18: 2 / 18: 2$ & 0.0 & 13.8 & 0.0 & 19.8 \\
697 & $18: 1 / 18: 2$ & 0.0 & 10.5 & 0.0 & 11.9 \\
699 & $18: 0 / 18: 2$ & 0.0 & 8.7 & 24.3 & 5.7 \\
701 & $18: 1 / 18: 1$ & 19.9 & 15.0 & 14.9 & 7.7 \\
\hline
\end{tabular}

Results are from 2 experiments, in which linoleate pretreatment was carried out for 6 or $24 \mathrm{~h}$ as indicated. Values have been normalised to an internal standard (PtdH 17:0/17:0) and are expressed as percentage mean ion abundance after correction for isotope contributions.

$\mathrm{m} / \mathrm{z}$ : mass to charge ratio

rodents has been extensively characterised using a euglycaemic-hyperinsulinaemic clamp [23, 28, 29]; such a diet causes significant insulin resistance in skeletal muscle, as well as a reduction in acute insulin-stimulated IRS-1 tyrosine phosphorylation [30]. In the present study, we verified by ipGTT that the mice fed a high-fat diet became glucoseintolerant relative to chow-fed controls (Fig. 5a). The high-fat diet caused a greater than $50 \%$ increase in the area under the blood glucose curves (Fig. 5b), consistent with muscle insulin resistance.

We then analysed the PtdH species present in skeletal muscle from mice fed a high-fat diet and from control mice, determining the molar content per $\mathrm{g}$ muscle of each form present by mass spectrometry. The results obtained resembled those from L6 myotubes pretreated with linoleate, in that certain PtdH species containing linoleate were significantly elevated by the high-fat diet (Table 2). Again, the novel species 18:2/18:2-PtdH was not detected in control muscle, but represented approximately $8 \%$ of the total PtdH in muscle from mice fed the high-fat diet. We also observed that $16: 0 / 18: 2-\mathrm{PtdH}$ increased from 12 to $23 \%$, although 18:1/18:2-PtdH did not change (Table 2). Reductions in the amounts of other PtdH molecular species were also seen, e.g. 16:0/16:0-, 16:0/18:1- and 18:0/18:1-PtdH, which had also fallen in L6 myotubes. The total amount of PtdH detected was reduced by $26 \%$ in muscle from mice on the high-fat diet, although it is possible that the concentrations of PtdH given in Table 2 were affected by lipid from other cell types such as adipocytes, even though visible fat had been carefully removed. There were some differences in the PtdH species found in skeletal muscle compared with L6 myotubes. 16:0/16:1-PtdH was not detected in skeletal muscle, whereas palmitoyl-arachidonyl (16:0/20:4)- and palmitoyl-docosahexaenoic (16:0/22:6)-PtdH had not been observed in L6 cells.
Specific inhibition of insulin-stimulated IRS-1 tyrosine phosphorylation by 18:2/18:2-PtdH Our data prompted the question of whether particular PtdH species were responsible for mediating the effects of linoleate on IRS-1 signalling and glucose disposal. We therefore treated L6 cells with PtdH species that had been indicated by mass spectrometry to be potential mediators in L6 myotubes and skeletal muscle (16:0/18:2- and 18:2/18:2-PtdH), as well as with certain species that were reduced in both models (16:0/ 16:0-, 16:0/18:1-PtdH) as controls. L6 cells were treated in medium supplemented with phosphatidylcholine micelles containing specific PtdH species. The cells were then stimulated with insulin and lysates analysed for tyrosinephosphorylated and total IRS-1. There was no difference in insulin-stimulated IRS-1 tyrosine phosphorylation in cells treated with micelles containing either no PtdH or 16:0/ 16:0-, 16:0/18:1- or 16:0/18:2-PtdH. However, cells pretreated with micelles containing 18:2/18:2-PtdH displayed a
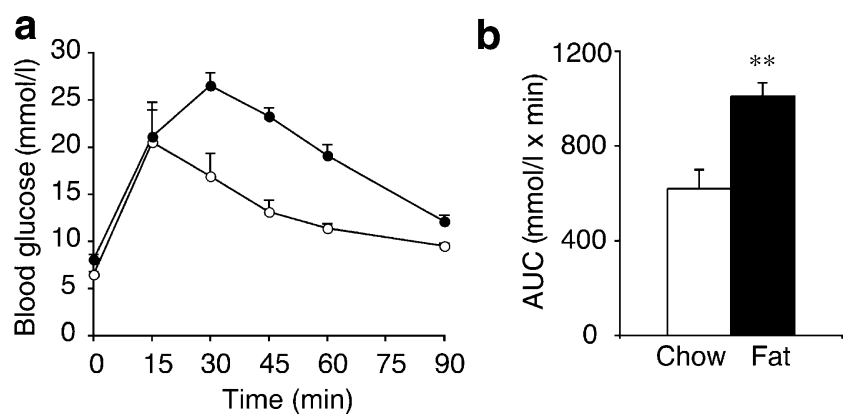

Fig. 5 Glucose tolerance in mice fed a high-fat diet rich in linoleate. a Mice were fed either a safflower oil-containing high-fat diet (filled circles) or a standard chow control diet (open circles) for 5 weeks and glucose tolerance was assessed. The mean blood glucose levels during the ipGTT are shown. b The mean blood glucose AUC is shown for chow-fed and high-fat-fed mice. ${ }^{* *} p<0.01$ for high-fat fed vs control ( $t$ test) ( $n=4$ per group) 
Table 2 Phosphatidic acid composition of skeletal muscle from mice fed chow or a high-fat diet

\begin{tabular}{llll}
\hline$m / z$ & Acyl composition & Chow (nmol/g tissue) & High-fat diet (nmol/g tissue) \\
\hline 647 & $16: 0 / 16: 0$ & $13.9 \pm 1.4$ & $6.2 \pm 1.3^{*}$ \\
671 & $16: 0 / 18: 2$ & $12.3 \pm 2.1$ & $22.8 \pm 1.9^{*}$ \\
673 & $16: 0 / 18: 1$ & $21.6 \pm 1.5$ & $9.1 \pm 2.1^{* *}$ \\
695 & $16: 0 / 20: 4$ & $15.4 \pm 1.3$ & $12.3 \pm 1.6$ \\
& $18: 2 / 18: 2$ & $0.0 \pm 0.0$ & $8.3 \pm 0.8^{* * *}$ \\
697 & $18: 1 / 18: 2$ & $8.4 \pm 2.0$ & $8.5 \pm 1.0$ \\
699 & $18: 1 / 18: 1$ & $12.2 \pm 1.6$ & $11.5 \pm 1.3$ \\
701 & $18: 0 / 18: 1$ & $15.8 \pm 2.3$ & $4.0 \pm 1.3^{* *}$ \\
719 & $16: 0 / 22: 6$ & $41.4 \pm 0.4$ & $21.0 \pm 2.6^{* * *}$ \\
\end{tabular}

Values have been normalised to an internal standard (PtdH 17:0/17:0), which was added at the time of tissue extraction and which was also used to calculate molar content.

$m / z$ : mass to charge ratio

${ }^{*} p<0.05$ for high-fat diet vs chow ( $n=4$ per group, by $t$ test).

$* * p<0.01$ for high-fat diet vs chow ( $n=4$ per group, by $t$ test).

$* * * p<0.001$ for high-fat diet vs chow ( $n=4$ per group, by $t$ test).

reduced level of IRS-1 tyrosine phosphorylation (Fig. 6a, b). Both 16:0/18:2- and 18:2/18:2-PtdH also caused a decrease in the total IRS-1 protein detected in these experiments (Fig. 6a, c). This is reminiscent of the effects of DAGKe, but also suggests that the inhibition of IRS-1 tyrosine phosphorylation cannot be simply explained by a loss of IRS-1.

We have previously shown that linoleate inhibits glycogen synthesis in cultured myotubes $[14,17]$. To determine whether 18:2/18:2-PtdH could also specifically mimic this aspect of linoleate-induced insulin resistance, we measured glycogen synthesis after cells had been pretreated in the absence or presence of PtdH-containing micelles. While micelles themselves reduced insulin-stimulated glycogen synthesis in the absence of any PtdH, only 18:2/18:2-PtdH was able to inhibit this further, by approximately $50 \%$ (Fig. 6d). The effects of 18:2/18:2-PtdH on IRS-1, as well as the demonstration of the inhibition of a metabolic endpoint of insulin action by the lipid, further support an inhibitory effect of linoleate-derived $\mathrm{PtdH}$ on insulin action and indicate a level of specificity dictated by the acyl side chains.

\section{Discussion}

We show here that the unsaturated NEFA linoleate induces insulin resistance in L6 myotubes at the level of IRS-1 tyrosine phosphorylation and its subsequent association with PI3K. In addition, we demonstrated that linoleate affects the intracellular $\mathrm{PtdH}$ profile and that direct modulation of PtdH can also affect IRS-1 phosphorylation. Further, our results are consistent with an inhibitory role for 18:2/18:2-PtdH, rather than for DAG and nPKCs.
The absence of a change in total DAG effected by linoleate pre-treatment, despite elevation of TG, is counterintuitive, but consistent with previous work [31, 32]. This and the fact that we were unable to show elevation of DAG in a specific compartment by subcellular fractionation suggests that the activation of $\mathrm{nPKC}$ isoforms is therefore likely to occur in response to elevation of minor polyunsaturated DAG species. Given the significant increase in TG content in linoleate-pretreated myotubes, the observed alterations in DAG and PtdH species upon lipid treatment probably reflect the role of these lipids as intermediates in de novo synthesis of $\mathrm{TG}$, rather than the activity of phospholipases, which can elevate DAG and PtdH acutely in a signalling context. Nevertheless, linoleate-induced changes in DAG were probably responsible for PKC activation in L6 cells because overexpression of DAGKe caused a reversal of the effects of linoleate on nPKC isoforms. Importantly, however, this was unable to prevent the inhibitory effect of linoleate on IRS-1 tyrosine phosphorylation. The lack of evidence that DAG plays a role in the insulin resistance seen in this model is in agreement with our previous findings, made using pharmacological PKC inhibitors and dominant-negative PKC mutants [18].

The effects of DAGKe overexpression and lisofylline pretreatment on IRS-1 tyrosine phosphorylation, taken together with the observations that $\mathrm{PtdH}$ turnover was increased in linoleate-treated myotubes, strongly support a role for PtdH itself in the generation of insulin resistance by unsaturated NEFAs. This notion was supported by mass spectrometry data, which indicated that L6 cells treated with linoleate favoured the incorporation of unsaturated 18:2 side chains into $\mathrm{PtdH}$, reflecting their increased availability. This induced the appearance of species such as 18:2/18:2-PtdH, which were undetected in control L6 
a
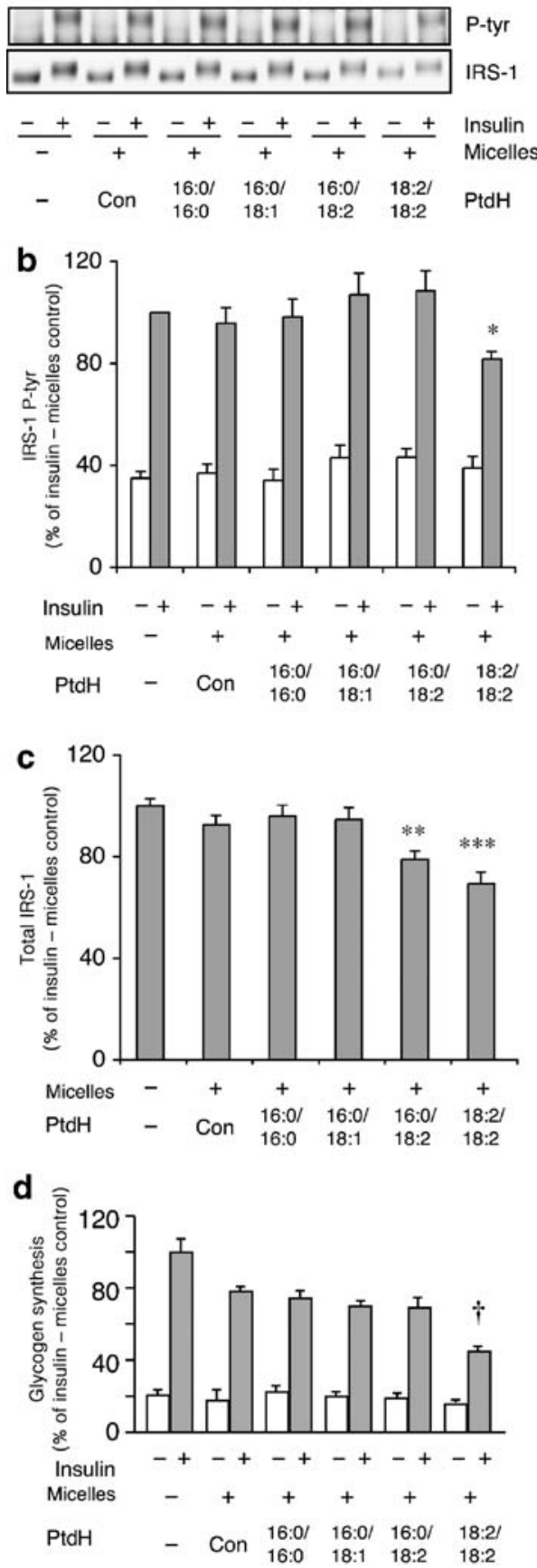

Fig. 6 Effects of different species of PtdH on IRS-1 tyrosine phosphorylation. a L6 cells were treated for $24 \mathrm{~h}$ without or with phosphatidylcholine micelles containing the long chain PtdH species as indicated. Cells were stimulated with insulin, lysates prepared and phosphotyrosine (P-tyr) and IRS-1 levels determined by immunoblot. A representative experiment of three experiments carried out in triplicate is shown. The results of densitometric analyses for phosphotyrosine (b) and total IRS-1 protein (c) are also shown. ${ }^{*} p<$ $0.05, * * p<0.01, * * * p<0.001$ for PtdH-containing micelles vs control micelles $(t$ test). d Cells were treated for a further $1 \mathrm{~h}$ with micelles in the absence or presence of insulin for the determination of glycogen synthesis. The combined data from two experiments carried out in triplicate are shown. $\dagger p<0.005$ for $18: 2 / 18: 2-\mathrm{PtdH}$ vs any other PtdH ( $t$ test) cells. Our data from mouse skeletal muscle indicated that certain of these species are in fact normally present in intact skeletal muscle, perhaps due to the presence of linoleate in standard chow. However, their levels were significantly elevated in mice fed a high-fat diet, again probably due to increased linoleate availability. A notable exception was the appearance of the novel species 18:2/18:2-PtdH, which had been undetectable in control muscle as well as in untreated L6 cells. The demonstration that only this particular PtdH species was able to inhibit insulin-stimulated IRS-1 tyrosine phosphorylation in L6 myotubes suggests that it may be an important mediator of insulin resistance.

Our experiments involving the use of DAGKe, lisofylline or PtdH-containing micelles indicated that IRS-1 protein levels as well as tyrosine phosphorylation could be affected by alterations in PtdH availability. However, changes in tyrosine phosphorylation did not always correspond with altered IRS-1 expression. For example, lisofylline increased IRS-1 levels in both control and linoleate-treated cells, but only restored phosphorylation in the presence of linoleate, rather than increasing it under all conditions. Furthermore, both 16:0/18:2- and 18:2/18:2PtdH micelles reduced IRS-1 levels, but only 18:2/18:2$\mathrm{PtdH}$ affected tyrosine phosphorylation. These findings suggest that the two effects are at least partly independent and a PtdH-induced mechanism affecting tyrosine phosphorylation, but not IRS-1 levels, may contribute to the diminished IRS-1 phosphorylation in linoleate-treated L6 cells and skeletal muscle [30]. A second mechanism affecting IRS-1 protein levels may additionally occur under harsher experimental conditions. Interestingly, L6 myotubes treated for prolonged periods with linoleate exhibit reduced IRS-1 levels (not shown), while morbidly obese patients have reduced IRS-1 abundance in skeletal muscle [33], suggesting that such a mechanism may become relevant upon greater lipid accumulation. An additional novel finding of the current study is the increase of IRS-1 protein levels effected by lisofylline. Although currently under evaluation for the treatment of autoimmunity and type 1 diabetes [34], the potential benefits of this drug for the treatment of insulin resistance and type 2 diabetes have not previously been reported.

Our previous investigations of lipid-treated myotubes demonstrated that unsaturated NEFAs such as oleate and linoleate can inhibit insulin signalling at the level of IRS-1/ PI3K, but have no effect on PKB and glycogen synthase kinase $3[14,17]$. Because these lipids are also able to inhibit insulin-stimulated glucose disposal in this model $[14,17]$, this may involve a PKB-independent link between IRS-1 and metabolic endpoints. Importantly, 18:2/18:2PtdH was also able inhibit insulin-stimulated glycogen synthesis in the current study, consistent with a role as a mediator of inhibition of insulin action by linoleate. While oleate is less potent than linoleate [14], the similarities 
between the effects of these lipids on myotubes suggest that oleate might also lead to accumulation of inhibitory PtdH species. Because oleate availability can lead to sparing of linoleate $\beta$-oxidation and hence accumulation of linoleatederived glycerolipids [35], it is conceivable that oleate could cause 18:2/18:2-PtdH accumulation, although this remains to be determined.

Although our models favour accumulation of linoleate, this lipid may still significantly affect glucose homeostasis upon increased availability of mixed lipids in a physiological setting. Compared with a hunter-gatherer diet, linoleate intake has increased approximately fourfold in the Western diet [36]. The insulin resistance caused by saturated and unsaturated NEFAs appears to be mediated by different lipid species (ceramide and PtdH respectively), which act at different sites of insulin signalling (PKB and IRS-1). This may result in the potentiation of inhibitory effects and a greater inhibition of glucose disposal.

While the mechanistic link between PtdH and the inhibition of IRS-1 tyrosine phosphorylation has not been investigated here, we speculate that activation of signalling proteins by the phospholipid may play a role [37]. For example, PtdH has been identified as an activator of mammalian target of rapamycin (mTOR) [38] and Raf-1 kinase [39], each of which are upstream of kinase cascades leading to IRS-1 serine phosphorylation [40, 41]. Alternatively, since PtdH is a key lipid involved in membrane biosynthesis and itself modulates membrane properties [42], the appearance of 18:2/18:2-PtdH may have indirect effects on signalling through changes in cell membrane composition. PtdH could also act at the level of gene expression [43]. Although linoleate itself is a peroxisome proliferator-activated receptor (PPAR) $\gamma$ agonist [44] and could promote the expression of enzymes involved in lipid synthesis to influence the availability of inhibitory intermediates, no evidence was observed for linoleate-induced upregulation of PPAR $\gamma$-dependent genes upon analysis of gene expression in L6 myotubes by transcript profiling using gene arrays or by RT-PCR (Cooney and Breivik, unpublished results). This suggests that PPAR $\gamma$ does not play a significant role in this model of insulin resistance.

In conclusion, we have shown that linoleate reduces IRS-1 tyrosine phosphorylation in L6 cells, which appears to be independent of DAG and nPKC function. Instead, we have indicated a role for PtdH and demonstrated that 18:2/ 18:2-PtdH, a novel species that is induced in linoleatetreated cells and in insulin-resistant muscle from mice on a high-fat diet, is selectively capable of inhibiting IRS-1 tyrosine phosphorylation. This mechanism may act in concert with others such as the ceramide-mediated inhibition of PKB induced by saturated NEFAs, depending on the NEFA profile of the diet. Finally, we have indicated inhibition of PtdH synthesis by LPAAT as a possible strategy for the treatment of lipid-induced insulin resistance in skeletal muscle.

Acknowledgements This work was funded by grants from the National Health and Medical Research Council (NH\&MRC) of Australia (to C. Schmitz-Peiffer and T. J. Biden) and Eli Lilly Australia (to C. Schmitz-Peiffer). R. Cazzolli was a recipient of an NH\&MRC Dora Lush Postgraduate Research Scholarship. N. Turner is supported by a NH\&MRC Peter Doherty Fellowship.

Duality of interest The authors declare that there is no duality of interest for this work.

\section{References}

1. DeFronzo RA, Gunnarsson R, Bjorkman O, Olsson M, Wahren J (1985) Effects of insulin on peripheral and splanchnic glucose metabolism in noninsulin-dependent (type II) diabetes mellitus. J Clin Invest 76:149-155

2. McGarry JD (2002) Banting lecture 2001: dysregulation of fatty acid metabolism in the etiology of type 2 diabetes. Diabetes 51:7-18

3. Nystrom FH, Quon MJ (1999) Insulin signalling: metabolic pathways and mechanisms for specificity. Cell Signal 11:563-574

4. Zick Y (2001) Insulin resistance: a phosphorylation-based uncoupling of insulin signaling. Trends Cell Biol 11:437-441

5. Schmitz-Peiffer C, Whitehead JP (2003) IRS-1 regulation in health and disease. IUBMB Life 55:367-374

6. Schmitz-Peiffer C (2000) Signalling aspects of insulin resistance in skeletal muscle: mechanisms induced by lipid oversupply. Cell Signal 12:583-594

7. Krebs M, Roden M (2005) Molecular mechanisms of lipidinduced insulin resistance in muscle, liver and vasculature. Diabetes Obes Metab 7:621-632

8. Schmitz-Peiffer C, Browne CL, Oakes ND et al (1997) Alterations in the expression and cellular localization of protein kinase $\mathrm{C}$ isozymes epsilon and theta are associated with insulin resistance in skeletal muscle of the high-fat-fed rat. Diabetes 46:169-178

9. Griffin ME, Marcucci MJ, Cline GW et al (1999) Free fatty acidinduced insulin resistance is associated with activation of protein kinase $\mathrm{C}$ theta and alterations in the insulin signaling cascade. Diabetes 48:1270-1274

10. Itani SI, Ruderman NB, Schmieder F, Boden G (2002) Lipidinduced insulin resistance in human muscle is associated with changes in diacylglycerol, protein kinase C, and IkappaB-alpha. Diabetes 51:2005-2011

11. Itani SI, Pories WJ, Macdonald KG, Dohm GL (2001) Increased protein kinase $\mathrm{C}$ theta in skeletal muscle of diabetic patients. Metabolism 50:553-557

12. Yu C, Chen Y, Cline GW et al (2002) Mechanism by which fatty acids inhibit insulin activation of insulin receptor substrate-1 (IRS-1)-associated phosphatidylinositol 3-kinase activity in muscle. J Biol Chem 277:50230-50236

13. Turinsky J, Bayly BP, O’Sullivan DM (1990) 1,2-Diacylglycerol and ceramide levels in rat skeletal muscle and liver in vivo. Studies with insulin, exercise, muscle denervation, and vasopressin. J Biol Chem 265:7933-7938

14. Schmitz-Peiffer C, Craig DL, Biden TJ (1999) Ceramide generation is sufficient to account for the inhibition of the insulin-stimulated $\mathrm{PKB}$ pathway in $\mathrm{C} 2 \mathrm{C} 12$ skeletal muscle cells pretreated with palmitate. J Biol Chem 274:24202-24210 
15. Cazzolli R, Carpenter L, Biden TJ, Schmitz-Peiffer C (2001) A role for protein phosphatase 2A-like activity, but not atypical protein kinase Czeta, in the inhibition of protein kinase B/Akt and glycogen synthesis by palmitate. Diabetes 50:2210-2218

16. Stratford S, Hoehn KL, Liu F, Summers SA (2004) Regulation of insulin action by ceramide: dual mechanisms linking ceramide accumulation to the inhibition of Akt/protein kinase B. J Biol Chem 279:36608-36615

17. Taylor AJ, Ye JM, Schmitz-Peiffer C (2006) Inhibition of glycogen synthesis by increased lipid availability is associated with subcellular redistribution of glycogen synthase. J Endocrinol 188:11-23

18. Cazzolli R, Craig DL, Biden TJ, Schmitz-Peiffer C (2002) Inhibition of glycogen synthesis by fatty acid in $\mathrm{C}(2) \mathrm{C}(12)$ muscle cells is independent of PKC-alpha, -epsilon, and -theta. Am J Physiol Endocrinol Metab 282:E1204-E1213

19. Nakamura K, Handa S (1984) Coomassie brilliant blue staining of lipids on thin-layer plates. Anal Biochem 142:406-410

20. Pol A, Martin S, Fernandez MA et al (2004) Dynamic and regulated association of caveolin with lipid bodies: modulation of lipid body motility and function by a dominant negative mutant. Mol Biol Cell 15:99-110

21. Whitehead JP, Simpson F, Hill MM et al (2004) Insulin and oleate promote translocation of inosine-5' monophosphate dehydrogenase to lipid bodies. Traffic 5:739-749

22. Nishijima M, Kuge O, Akamatsu Y (1986) Phosphatidylserine biosynthesis in cultured Chinese hamster ovary cells. I. Inhibition of de novo phosphatidylserine biosynthesis by exogenous phosphatidylserine and its efficient incorporation. J Biol Chem 261:5784-5789

23. Storlien LH, James DE, Burleigh KM, Chisholm DJ, Kraegen EW (1986) Fat feeding causes widespread in vivo insulin resistance, decreased energy expenditure and obesity in rats. Am J Physiol 251:E576-E583

24. Cooney GJ, Lyons RJ, Crew AJ et al (2004) Improved glucose homeostasis and enhanced insulin signalling in Grb14-deficient mice. EMBO J 23:582-593

25. Murphy DJ, Vance J (1999) Mechanisms of lipid-body formation. Trends Biochem Sci 24:109-115

26. Pettitt TR, Wakelam M (1999) Diacylglycerol kinase epsilon, but not zeta, selectively removes polyunsaturated diacylglycerol, inducing altered protein kinase $\mathrm{C}$ distribution in vivo. J Biol Chem 274:36181-36186

27. Miles K, Wagner M (2000) Overexpression of nPKC theta is permissive for myogenic differentiation. J Cell Biochem 79:71-79

28. Kraegen EW, James DE, Storlien LH, Burleigh KM, Chisholm DJ (1986) In vivo insulin resistance in individual peripheral tissues of the high fat fed rat: assessment by euglycemic clamp plus deoxyglucose administration. Diabetologia 29:192-198

29. Kraegen EW, Clark PW, Jenkins AB, Daley EA, Chisholm DJ, Storlien LH (1991) Development of muscle insulin resistance after liver insulin resistance in high-fat-fed rats. Diabetes 40:1397-1403
30. Frangioudakis G, Ye JM, Cooney GJ (2005) Both saturated and n6 polyunsaturated fat diets reduce phosphorylation of insulin receptor substrate-1 and protein kinase B in muscle during the initial stages of in vivo insulin stimulation. Endocrinology 146: 5596-5603

31. Lu X, Yang XY, Howard RL, Walsh JP (2000) Fatty acids modulate protein kinase $\mathrm{C}$ activation in porcine vascular smooth muscle cells independently of their effect on de novo diacylglycerol synthesis. Diabetologia 43:1136-1144

32. Montell E, Turini M, Marotta M et al (2001) DAG accumulation from saturated fatty acids desensitizes insulin stimulation of glucose uptake in muscle cells. Am J Physiol Endocrinol Metab 280:E229-E237

33. Goodyear LJ, Giorgino F, Sherman LA, Carey J, Smith RJ, Dohm GL (1995) Insulin receptor phosphorylation, insulin receptor substrate-1 phosphorylation, and phosphatidylinositol 3-kinase activity are decreased in intact skeletal muscle strips from obese subjects. J Clin Invest 95:2195-2204

34. Yang Z, Chen M, Nadler JL (2005) Lisofylline: a potential lead for the treatment of diabetes. Biochem Pharmacol 69:1-5

35. Emmison N, Gallagher PA, Coleman RA (1995) Linoleic and linolenic acids are selectively secreted in triacylglycerol by hepatocytes from neonatal rats. Am J Physiol 269:R80-R86

36. Simopoulos AP (1994) Is insulin resistance influenced by dietary linoleic acid and trans fatty acids? Free Radic Biol Med 17:367372

37. Hodgkin MN, Pettitt TR, Martin A, Michell RH, Pemberton AJ, Wakelam MJ (1998) Diacylglycerols and phosphatidates: which molecular species are intracellular messengers? Trends Biochem Sci 23:200-204

38. Fang Y, Vilella-Bach M, Bachmann R, Flanigan A, Chen J (2001) Phosphatidic acid-mediated mitogenic activation of mTOR signaling. Science 294:1942-1945

39. Andresen BT, Rizzo MA, Shome K, Romero G (2002) The role of phosphatidic acid in the regulation of the Ras/MEK/Erk signaling cascade. FEBS Lett 531:65-68

40. Zick Y (2005) Ser/Thr phosphorylation of IRS proteins: a molecular basis for insulin resistance. Sci STKE 2005(268):pe4

41. De Fea K, Roth RA (1997) Modulation of insulin receptor substrate-1 tyrosine phosphorylation and function by mitogenactivated protein kinase. J Biol Chem 272:31400-31406

42. Wang XM, Devalah SP, Zhang WH, Welti R (2006) Signaling functions of phosphatidic acid. Prog Lipid Res 45:250-278

43. Loewen CJ, Gaspar ML, Jesch SA et al (2004) Phospholipid metabolism regulated by a transcription factor sensing phosphatidic acid. Science 304:1644-1647

44. Kliewer SA, Sundseth SS, Jones SA et al (1997) Fatty acids and eicosanoids regulate gene expression through direct interactions with peroxisome proliferator-activated receptors alpha and gamma. Proc Natl Acad Sci USA 94:4318-4323 